\title{
OPTICAL COUNTERPART OF THE LMXB GX 13+1
}

\author{
P. J. GROOT ${ }^{1}$, M. VAN DER KLIS ${ }^{1}$, J. VAN PARADIJS ${ }^{1,2}$, \\ T. AUGUSTEIJN ${ }^{3}$, M. BERGER ${ }^{1}$ \\ 1. Astronomical Institute 'Anton Pannekoek'/CHEAF, \\ University of Amsterdam, Amsterdam, The Netherlands \\ 2. Physics Department, University of Alabama, Alabama, USA \\ 3. ESO, La Silla, Chile
}

We have observed the IR counterpart of the Atoll type LMXB GX 13+1, (Naylor, Charles \& Longmore 1991), from 1993 July 11...30, with the Dutch $90 \mathrm{~cm}$ telescope at ESO using a Gunn $z(0.9-1.0 \mu \mathrm{m})$ filter. Over this period 110 usable images (integration time $12 \mathrm{~m}$ ) were obtained. The data were reduced using the ESO-MIDAS/DAPHOT reduction package. A Lomb-Scargle period search was performed with the PERIOD package.

Fig. 1 shows our Gunn $z$ band image with stars labeled as in Naylor et al. (1991). They propose their star 101 as the IR counterpart. Fig. 2 shows the light curve we obtained for this star. The star is clearly variable on a time-scale of $\sim 10 \mathrm{~d}$. To estimate any period more accurately we have made a Lomb-Scargle statistics periodigram (see Fig. 3); maximum power is at $12.6 \mathrm{~d}$. We estimate the error to be $\sim 1$ day.

The time interval covered by our observations (18d) is too short to establish the periodicity of the variation. If the orbital period is long as is suggested by our results it may be difficult to understand the difference between Atoll and $\mathrm{Z}$ sources on the basis of simple evolutionary arguments (see e.g. Lewin, van Paradijs \& van der Klis 1988).

Acknowledgements. We thank Jeroen de Jong, Vik Dhillon and Gene Magnier for the use of their programs.

\section{References}

Naylor, T., Charles, P.A., Longmore, A.J., 1991, MNRAS, 252, 203

Lewin, H.G., van Paradijs, J., van der Klis, M., 1988, Sp. Sc. Rev., 46, 273

367

A. Evans and J. H. Wood (eds.), Cataclysmic Variables and Related Objects, 367-368.

(C) 1996 Kluwer Academic Publishers. Printed in the Netherlands. 


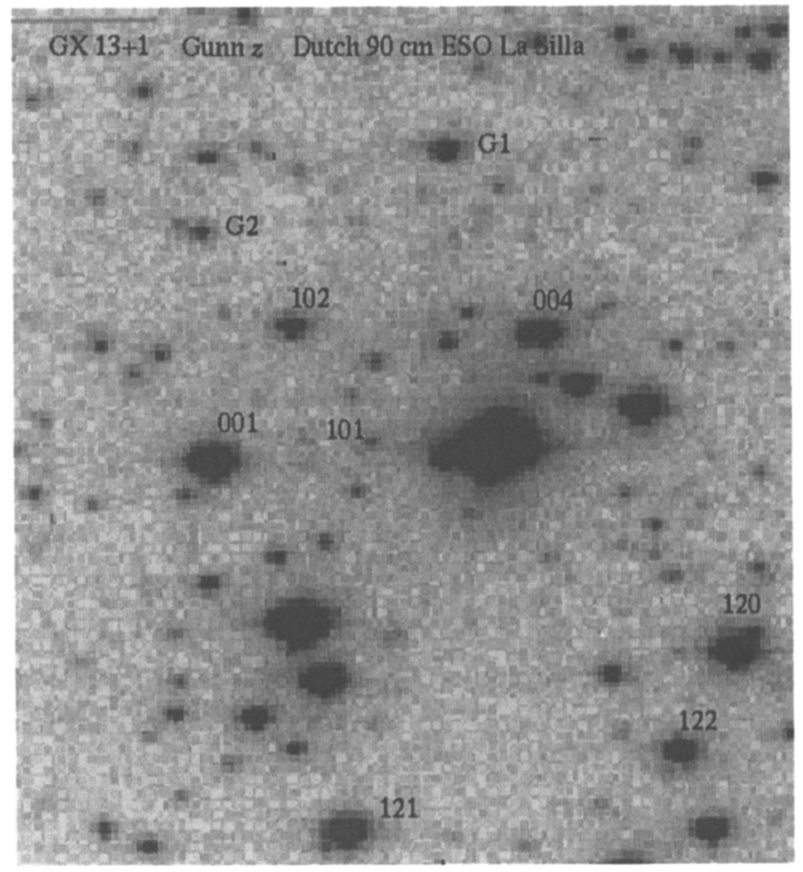

Figure 1. Gunn $z$ band image of GX $13+1$. Star 101 is the IR counterpart.
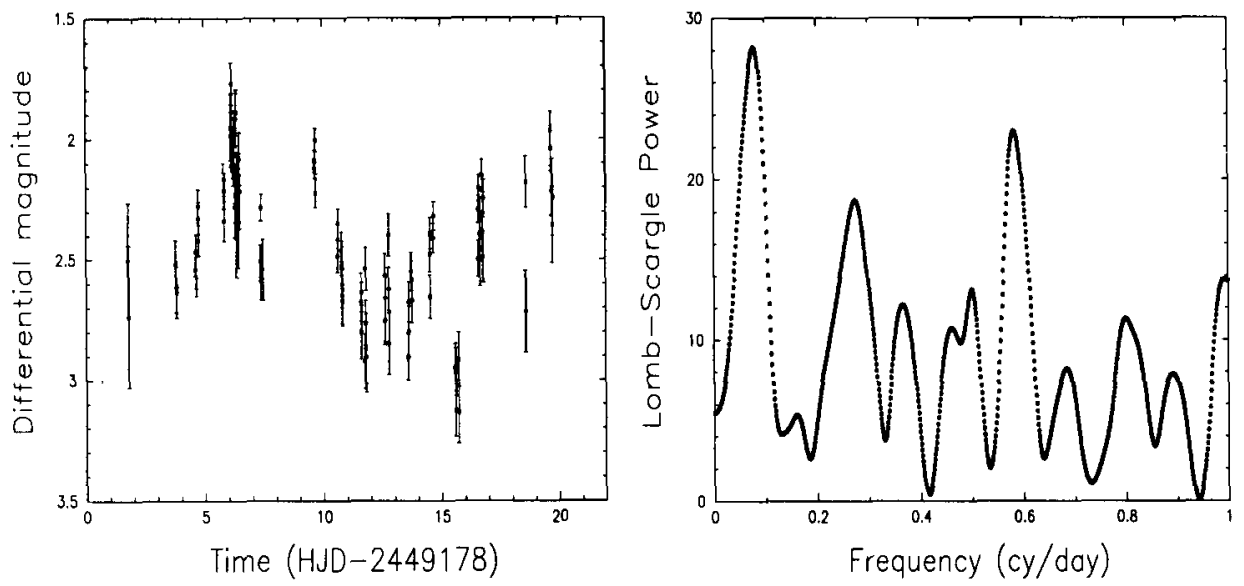

Figure 2. Light curve of star 101 in Fig. 1. Figure 3. Lomb-Scargle statistics power Magnitudes in units of average magnitude spectrum of the light curve in Fig. 2. of comparison stars. 